\title{
Multiplicative Dirac system
}

\author{
Tuba Gulsen ${ }^{1}$, Emrah Yilmaz ${ }^{1, *}$, Sertac Goktas ${ }^{2}$ \\ ${ }^{1}$ Dept. of Mathematics, Firat University, Elazıǵ, Turkey \\ ${ }^{2}$ Dept. of Mathematics, Mersin University, Mersin, Turkey \\ *Corresponding author: emrah231983@gmail.com
}

\begin{abstract}
We define a Dirac system in multiplicative calculus by some algebraic st ructures. Asymptotic estimates for eigenfunctions of the multiplicative Dirac system are obtained. Eventually, some fundamental properties of the multiplicative Dirac system are examined in detail.
\end{abstract}

Keywords: Dirac system; multiplicative calculus, Eigenvalue, Eigenfunction, simplicity.

\section{Introduction}

Multiplicative calculus was introduced by Grossman \& Katz (Grossman, 1979; Grossman \& Katz, 1972) in 1967 as an alternative to classical calculus. This type of calculus is also known as nonNewtonian because of its difference from classical calculus of Newton and Leibniz. Multiplicative calculus is a useful supplement to classical calculus in that it is tailored to situations involving exponential functions in the same sense that classical calculus is tailored to situations involving linear functions. Multiplicative calculus moves the roles of subtraction and addition to division and multiplication. There are actually many reasons to study multiplication analysis. It improves the work of additive calculations indirectly. Problems that are difficult to solve in the classical case c an be solved with incredible ease in here. Every property in the Newtonian case can be defined in multiplicative analysis within certain rules.

Many events in nature change exponentially. For example, the populations of countries, the magnitude of an earthquake (Benford, 1938) are events that behave in this manner. For this reason, using multiplicative analysis instead of classical analysis allows a better physical evaluation of these types of events. This form of calculus also provides better results than the classical case in many fields such as finance, e conomics, biology and d e mographics. A very limited number of studies have been conducted on this analysis until the beginning of the 2000s. Recently, various studies have been carried out on it and quality and effective results have been obtained (see (Akkus \& Kizilaslan, 2019; Bashirov \& Riza, 2011; Bashirov et al., 2011; Boruah \& Hazarika, 2018; Florack \& Assen, 2012; Guenther, 1983; Slavík, 2007; Stanley, 1999; Yazlik et al., 2016)).

As known, a Dirac system is an efficient e quation s ystem in $\mathrm{m}$ athematical physics in the classical case. A Dirac system, which contributes to the formulation of quantum mechanics for elementary particles with half a spin such as electrons, was proposed by Dirac to eliminate some of the problems in the Klein-Gordon equation (Dirac, 1928). The non-relativistic Schrödinger equation is not sufficient to explain experiments performed at energies much higher than the rest mass energy. For this, the solutions of the Dirac equation, one of the relativistic wave equations, are required. For all these reasons, the Dirac equation system has a very important place in quantum physics. Many studies have been carried out over the years on this system and many important results have been obtained for the classical case (see (Allahverdiev \& Tuna, 2017; Bairamov et al., 2010; Gasymov \& Levitan, 1966; Joa \& Minkin, 1997; Keskin \& Ozkan, 2011; Levitan \& Sargsjan, 1991; Çöl \& Mamedov, 2012; Moses, 1956; Prats \& Toll, 1959; Sharma et al., 2011; Thaller, 1992; Verde, 1959; Weiss et al., 1972)). Hence, we want to examine a multiplicative Dirac system other than Newtonian calculus. Before moving on to the 
basic results, let us firstly express the concepts and important theorems of multiplicative analysis that we will use in our study.

Definition 1.1. (Bashirov et al., 2008) Let $f: A \subset \mathbb{R} \rightarrow \mathbb{R}$ be differentiable in usual case and $f(t)>0$ for all $t$. If the below limit exists and positive

$$
f^{*}(t)=\lim _{h \rightarrow 0}\left[\frac{f(t+h)}{f(t)}\right]^{\frac{1}{h}},
$$

$f^{*}(t)$ is called ${ }^{*}$ derivative of $f$ at $t$.

Lemma 1.2. (Bashirov et al., 2008) Let $f: A \rightarrow \mathbb{R}$ be positive and classical differentiable at $t$. Then, there is following relation between classical and ${ }^{*}$ derivatives.

$$
f^{*}(t)=e^{(\ln o f)^{\prime}(t)}
$$

Theorem 1.3. (Bashirov et al., 2008) Let $f, h$ be ${ }^{*}$ differentiable and $p$ be classical differentiable at $t$. The following equalities hold for * derivative.

i. $(c f)^{*}(t)=f^{*}(t)$,

ii. $(f h)^{*}(t)=f^{*}(t) h^{*}(t)$,

iii. $(f / h)^{*}(t)=f^{*}(t) / h^{*}(t)$

$i v \cdot\left(f^{p}\right)^{*}(t)=f^{*}(t)^{p(t)} f(t)^{p^{\prime}(t)}$,

$v \cdot(f o p)^{*}(t)=f^{*}(p(t))^{p^{\prime}(t)}$,

vi. $(f+h)^{*}(t)=f^{*}(t)^{\frac{f(t)}{f(t)+h(t)}} h^{*}(t)^{\frac{h(t)}{f(t)+h(t)}}$,

where $c$ is a positive constant.

The system of equations we will establish in our study will include the multiplicative derivative defined above. Since the multiplicative integral will emerge while obtaining the eigenfunctions of the multiplicative Dirac system, let us express the notion and basic properties of multiplicative integration.

Definition 1.4. (Bashirov et al., 2008) Let $f$ be a positive bounded function on $[a, b]$ where $-\infty<$ $a<b<\infty$. Consider the partition $\mathcal{P}=\left\{x_{0}, x_{1}, \ldots, x_{n}\right\}$ of $[a, b]$ and the numbers $\xi_{1}, \xi_{2}, \ldots, \xi_{n}$ associated with the partition $\mathcal{P} . f$ is said to be integrable in the multiplicative sense or *integrable if there exists a number $\mathbf{P}$ having the property: for every $\varepsilon>0$ there exists a partition $\mathcal{P}_{\varepsilon}$ of $[a, b]$ such that $|\mathbf{P}(f, \mathcal{P})-\mathbf{P}|<\varepsilon$ for every refinement $\mathcal{P}$ of $\mathcal{P}_{\varepsilon}$ independently on selection of the numbers associated with the partition $\mathcal{P}$ where

$$
\mathbf{P}(f, \mathcal{P})=\prod_{i=1}^{n} f\left(\xi_{i}\right)^{\left(x_{i}-x_{i-1}\right)}
$$

Then, the symbol $\int_{a}^{b} f(x)^{d x}$ denotes multiplicative integral or *integral of $f$ on $[a, b]$. By this definition, if $f$ is positive and Riemann integrable on $[a, b]$, then it is *integrable on $[a, b]$ and

$$
\int_{a}^{b} f(x)^{d x}=e^{\int^{b}(\ln o f)(x) d x} .
$$

Conversely, one can show that if $f$ is Riemann integrable on $[a, b]$, then

$$
\int_{a}^{b} f(x) d x=\ln \int_{a}^{b}\left(e^{f(x)}\right)^{d x} .
$$


Theorem 1.5. (Bashirov et al., 2008) Let $f, h$ be positive bounded functions on $[a, b]$ where $-\infty<$ $a<b<\infty$. If $f, h$ are ${ }^{*}$ integrable on $[a, b]$, the following expressions hold:

$$
\begin{aligned}
& i \cdot \int_{a}^{b}\left[f(x)^{p}\right]^{d x}=\left[\int_{a}^{b} f(x)^{d x}\right]^{p}, \quad p \in \mathbb{R}, \\
& \text { ii. } \int_{a}^{b}[f(x) h(x)]^{d x}=\int_{a}^{b} f(x)^{d x} \int_{a}^{b} h(x)^{d x}, \\
& \text { iii. } \int_{a}^{b}\left[\frac{f(x)}{h(x)}\right]^{d x}=\frac{\int_{a}^{b} f(x)^{d x}}{\int_{a}^{b} h(x)^{d x}}, \\
& \text { iv. } \int_{a}^{b} f(x)^{d x}=\int_{a}^{c} f(x)^{d x} \int_{c}^{b} f(x)^{d x}, \quad a \leq c \leq b .
\end{aligned}
$$

The main focus of this study is to redefine the classical Dirac system in the multiplicative case. The Dirac system will be considered from a different perspective and the results will be compared in classical and multiplicative calculi. In the following years, the theory of complex functions in multiplicative analysis which is necessary especially while dealing with multiplicative analysis in spectral theory was defined by (Bashirov \& Norozpur, 2018). This is required in difficult applications of the Dirac system. The rest of this study is organized as follows: In the second section, we construct a multiplicative Dirac system by arithmetic operations. The asymptotic estimates for the eigenfunction of the multiplicative Dirac system is established. In the last section, some spectral properties of the multiplicative Dirac system are examined.

\section{Multiplicative Dirac system}

In this section, a multiplicative Dirac system is established by some algebraic structures and the eigenfunctions of the constructed problem are obtained. Accordingly, let us express multiplicative algebraic structures that we will encounter while establishing and solving the multiplicative Dirac system. Arithmetic operations created with exponential functions are called multiplicative algebraic operations. Let's show some properties of these operations with a multiplicative arithmetic table for $x, y \in \mathbb{R}^{+}$.

$$
x \ominus y=\frac{x}{y}, \quad x \oplus y=x y, \quad x \odot y=x^{\ln y}=y^{\ln x} .
$$

These operations create some algebraic structures. If $\oplus: A \times A \rightarrow A$ is an operation where $A \neq \phi$ and $A \subset \mathbb{R}^{+}$, the algebraic structure $(A, \oplus)$ is called multiplicative group. Similarly, $(A, \oplus, \odot)$ is a multiplicative ring. This situation gives us the opportunity to use these processes easily and define different structures. Also, multiplicative Dirac system includes multiplicative matrices. We overcome many problems using the basic properties of these multiplicative matrices (Kadak and Gurefe, 2016; Gurefe et al., 2016).

Let us consider the below multiplicative Dirac system

$$
L[y]=\left(B \odot y^{*}(t)\right) \oplus(Q(t) \odot y(t))=e^{\lambda} \odot y(t), \quad t \in[a, b],
$$

with the conditions

$$
\begin{aligned}
\left(e^{\alpha} \odot y_{1}(a)\right) \oplus\left(e^{\beta} \odot y_{2}(a)\right) & =1 \\
\left(e^{\gamma} \odot y_{1}(b)\right) \oplus\left(e^{\delta} \odot y_{2}(b)\right) & =1,
\end{aligned}
$$

where $\left(\alpha^{2}+\beta^{2}\right)\left(\gamma^{2}+\delta^{2}\right) \neq 1$ and

$$
B=\left[\begin{array}{cc}
1 & e \\
e^{-1} & 1
\end{array}\right], \quad Q(t)=\left[\begin{array}{cc}
e^{q(t)} & 1 \\
1 & e^{r(t)}
\end{array}\right]
$$


We assume that $a=1, b=e$ throughout this study without loss of generality. In fact, $[a, b]$ is mapped to $[1, e]$ by means of the substitution $x=\left(\frac{t}{a}\right)^{\frac{1}{\ln \frac{b}{a}}}$. Here, $\lambda$ is a spectral parameter and $q, r$ are real-valued, positive and *integrable functions. For the above system

$$
y(t, \lambda)=\left[\begin{array}{ll}
y_{1}(t, \lambda) & y_{2}(t, \lambda)
\end{array}\right]^{T}
$$

is the eigenfunction of the problem (1)-(2) corresponding to the eigenvalue $\lambda$ where $T$ denotes transpose of the matrix and $y_{j}, j=1,2$ are continuous and positive functions. If we expand and simplify this system by using the properties of multiplicative calculus, the following multiplicative system is obtained.

$$
\begin{aligned}
y_{2}^{*} y_{1}^{q(t)} & =y_{1}^{\lambda}, \\
y_{1}^{*} y_{2}^{-r(t)} & =y_{2}^{-\lambda},
\end{aligned}
$$

with the conditions

$$
\begin{gathered}
y_{1}^{\alpha}(a) y_{2}^{\beta}(a)=1 \\
y_{1}^{\gamma}(b) y_{2}^{\delta}(b)=1
\end{gathered}
$$

In order to avoid any difficulties in expressing the main parts of the study, the inner product will be defined and the spaces used throughout the study will be given in the multiplicative case.

Definition 2.1. Let $X$ be a non empty set and $<,>_{*}: X \times X \rightarrow \mathbb{R}^{+}$be a function such that the below axioms hold for all $x, y, z \in X$

M1) $<x, x>_{*} \geq 1$,

M2) $<x, x>_{*}=1$ if and only if $x=1$,

M3) $<x \oplus y, z>_{*}=<x, z>_{*} \oplus<y, z>_{*}$,

M4) $<e^{\alpha} \odot x, y>_{*}=e^{\alpha} \odot<x, y>_{*}, \alpha \in \mathbb{R}$

M5) $<x, y>_{*}=<y, x>_{*}$.

Then, $\left(X,<,>_{*}\right)$ is called $*^{*}$ inner product space and $<,>_{*}$ is *inner product on $X$.

Lemma 2.2. The space

$$
L_{2}^{*}[a, b]=\left\{f: \int_{a}^{b}[f(x) \odot f(x)]^{d x}<\infty\right\}
$$

is an *inner product space with

$$
\begin{aligned}
& <,>_{*}: L_{2}^{*}[a, b] \times L_{2}^{*}[a, b] \rightarrow \mathbb{R}^{+}, \\
& <f, h>_{*}=\int_{a}^{b}[f(x) \odot h(x)]^{d x},
\end{aligned}
$$

where $f, h \in L_{2}^{*}[a, b]$ are positive functions.

Proof: The proof can be easily demonstrated using the properties of the multiplicative inner product and the definition of the given space.

Lemma 2.3. (Kadak and Gurefe, 2016) Let $f:[a, b] \subset \mathbb{R} \rightarrow \mathbb{R}$ be ${ }^{*}$ integrable, positive function and $h:[a, b] \subset \mathbb{R} \rightarrow \mathbb{R}$ be differentiable, positive function. Then,

$$
\int_{a}^{b}\left[f^{*}(x)^{h(x)}\right]^{d x}=\frac{f(b)^{h(b)}}{f(a)^{h(a)}} \frac{1}{\int_{a}^{b}\left[f(x)^{h^{\prime}(x)}\right]^{d x}}
$$


This expression is known as *integration by parts formula. This lemma will have an important place in the proof of the next theorem.

Theorem 2.4. The eigenfunction of the problem (1)-(2) have the following asymptotic estimates

$$
\begin{aligned}
& y_{1}(t, \lambda)=\left[y_{2}(1)\right]^{-\sin \lambda(t-1)} \oplus\left[y_{1}(1)\right]^{\cos \lambda(t-1)} \oplus \int_{1}^{t}\left[\left(y_{1}^{q(s)}\right)^{\sin \lambda(t-s)} \oplus\left(y_{2}^{r(s)}\right)^{\cos \lambda(t-s)}\right]^{d s}, \\
& y_{2}(t, \lambda)=\left[y_{1}(1)\right]^{\sin \lambda(t-1)} \oplus\left[y_{2}(1)\right]^{\cos \lambda(t-1)} \oplus \int_{1}^{t}\left[\left(y_{1}^{q(s)}\right)^{-\cos \lambda(t-s)} \oplus\left(y_{2}^{r(s)}\right)^{\sin \lambda(t-s)}\right]^{d s},
\end{aligned}
$$

where $y(t, \lambda)=\left[\begin{array}{ll}y_{1}(t, \lambda) & y_{2}(t, \lambda)\end{array}\right]^{T}$.

Proof. By the given multiplicative Dirac system,

$$
\begin{aligned}
\int_{1}^{t}\left[\left(y_{1}^{q(s)}\right)^{\sin \lambda(t-s)} \oplus\left(y_{2}^{r(s)}\right)^{\cos \lambda(t-s)}\right]^{d s} & =e^{\int^{t} \ln \left[\left(y_{1}^{q(s)}\right)^{\sin \lambda(t-s)}\left(y_{2}^{r(s)}\right)^{\cos \lambda(t-s)}\right] d s} \\
& =e^{\int^{t}\left[\ln y_{1}^{q(s)} \sin \lambda(t-s)+\ln y_{2}^{r(s)} \cos \lambda(t-s)\right] d s .} .
\end{aligned}
$$

Then, after some computations,

$$
\begin{aligned}
\int_{1}^{t}\left[\left(y_{1}^{q(s)}\right)^{\sin \lambda(t-s)} \oplus\left(y_{2}^{r(s)}\right)^{\cos \lambda(t-s)}\right]^{d s} & =e^{\int^{t} \frac{-y_{2}^{\prime}}{y_{2}} \sin \lambda(t-s) d s} e^{\lambda \int_{1}^{t} \ln y_{1} \sin \lambda(t-s) d s} \\
& =e^{\int^{t} \frac{y_{1}^{\prime}}{y_{1}} \cos \lambda(t-s) d s} e^{\lambda \int_{1}^{t} \ln y_{2} \cos \lambda(t-s) d s} .
\end{aligned}
$$

Then, integration by parts method in multiplicative case yields

$$
\begin{gathered}
e^{\int^{t} \frac{-y_{2}^{\prime}}{y_{2}} \sin \lambda(t-s) d s}=\frac{\left[y_{2}(1)\right]^{\sin \lambda(t-1)}}{e^{\lambda \int_{1}^{t} \ln y_{2} \cos \lambda(t-s) d s},} \\
e^{\int^{t} \frac{y_{1}^{\prime}}{y_{1}} \cos \lambda(t-s) d s}=\frac{y_{1}(t)\left[y_{1}(1)\right]^{-\cos \lambda(t-1)}}{e^{\lambda \int_{1}^{t} \ln y_{1} \sin \lambda(t-s) d s}} .
\end{gathered}
$$

By considering (4) and (5) in (3), we get

$$
\int_{1}^{t}\left[\left(y_{1}^{q(s)}\right)^{\sin \lambda(t-s)} \oplus\left(y_{2}^{r(s)}\right)^{\cos \lambda(t-s)}\right]^{d s}=\frac{1}{\left[y_{2}(1)\right]^{-\sin \lambda(t-1)}} \frac{y_{1}(t)}{\left[y_{1}(1)\right]^{\cos \lambda(t-1)}},
$$

and

$$
y_{1}(t, \lambda)=\left[y_{2}(1)\right]^{-\sin \lambda(t-1)} \oplus\left[y_{1}(1)\right]^{\cos \lambda(t-1)} \oplus \int_{1}^{t}\left[\left(y_{1}^{q(s)}\right)^{\sin \lambda(t-s)} \oplus\left(y_{2}^{r(s)}\right)^{\cos \lambda(t-s)}\right]^{d s} .
$$

Similarly, the second component of eigenfunction $y(t, \lambda)$ can be obtained in the same way. It completes the proof.

The difference between the results obtained in the classical case and these results will create some important differences in quantum physics. In the next section, some theorems that have a very important place in explaining the physical properties of this system in relation to eigenfunctions are expressed and proven. 


\section{Some spectral properties of multiplicative Dirac system}

In this section, we examine some properties of multiplicative Dirac system as reality, simplicity, orthogonality and self-adjointness.

Lemma 3.1. The eigenfunctions $u(t)=\left[\begin{array}{ll}u_{1}(t) & u_{2}(t)\end{array}\right]^{T}$ and $v(t)=\left[\begin{array}{ll}v_{1}(t) & v_{2}(t)\end{array}\right]^{T}$ corresponding to the distinct eigenvalues $\lambda_{1}$ and $\lambda_{2}$ are orthogonal, i.e.

$$
\int_{a}^{b}\left[u^{T}\left(t, \lambda_{1}\right) \odot v\left(t, \lambda_{2}\right)\right]^{d t}=1 .
$$

Proof: The below equations can be written according to the definition of eigenfunctions

$$
\begin{aligned}
u_{2}^{*} u_{1}^{q(t)} & =u_{1}^{\lambda_{1}}, \\
u_{1}^{*} u_{2}^{-r(t)} & =u_{2}^{-\lambda_{1}},
\end{aligned}
$$

and

$$
\begin{aligned}
v_{2}^{*} v_{1}^{q(t)} & =v_{1}^{\lambda_{2}}, \\
v_{1}^{*} v_{2}^{-r(t)} & =v_{2}^{-\lambda_{2}} .
\end{aligned}
$$

Here, if circular sum and circular product operations are taken into account and applied to the above equations, we get

$$
\frac{\left(u_{2}^{*}\right)^{\ln v_{1}} u_{1}^{q(t) \ln v_{1}}\left(v_{1}^{*}\right)^{\ln u_{2}} v_{2}^{-r(t) \ln u_{2}}}{\left(u_{1}^{*}\right)^{\ln v_{2}} u_{2}^{-r(t) \ln v_{2}}\left(v_{2}^{*}\right)^{\ln u_{1}} v_{1}^{q(t) \ln u_{1}}}=\left(u_{1}^{\ln v_{1}} u_{2}^{\ln v_{2}}\right)^{\lambda_{1}-\lambda_{2}} .
$$

If we continue to use and edit the same properties,

$$
\left[\left(u_{2}^{*} \odot v_{1}\right) \ominus\left(u_{1}^{*} \odot v_{2}\right)\right] \oplus\left[\left(v_{1}^{*} \odot u_{2}\right) \ominus\left(v_{2}^{*} \odot u_{1}\right)\right]=\left[\left(u_{1} \odot v_{1}\right) \oplus\left(u_{2} \odot v_{2}\right)\right]^{\lambda_{1}-\lambda_{2}},
$$

and

$$
\left[\left(u_{2} \odot v_{1}\right) \ominus\left(u_{1} \odot v_{2}\right)\right]^{*}=\left[\left(u_{1} \odot v_{1}\right) \oplus\left(u_{2} \odot v_{2}\right)\right]^{\lambda_{1}-\lambda_{2}},
$$

are obtained. Now let's make the proof of this last equation with multiplicative techniques.

$$
\begin{aligned}
{\left[\left(u_{2} \odot v_{1}\right) \ominus\left(u_{1} \odot v_{2}\right)\right]^{*}=\left(\frac{u_{2} \odot v_{1}}{u_{1} \odot v_{2}}\right)^{*} } & =\frac{\left(u_{2}^{*}\right)^{\ln v_{1}} u_{2}^{\frac{v_{1}^{\prime}}{v_{1}}}}{\left(u_{1}^{*}\right)^{\ln v_{2}} u_{2}^{v_{2}^{\prime}}} \\
& =\frac{\left(u_{1}^{\lambda_{1}-q(t)}\right)^{\ln v_{1}} u_{2}^{\ln v_{2}^{r(t)-\lambda_{2}}}}{\left(u_{2}^{r(t)-\lambda_{1}}\right)^{\ln v_{2}} u_{1}^{\ln v_{1}^{\lambda_{2}-q(t)}}} \\
& =\left[\left(u_{1} \odot v_{1}\right) \oplus\left(u_{2} \odot v_{2}\right)\right]^{\lambda_{1}-\lambda_{2}} .
\end{aligned}
$$

Therefore, by taking *integral for both sides of (6) on $[1, e]$, we get

$$
\int_{1}^{e}\left\{\left[\left(u_{1} \odot v_{1}\right) \oplus\left(u_{2} \odot v_{2}\right)\right]^{\lambda_{1}-\lambda_{2}}\right\}^{d t}=\frac{\left[\left(u_{2} \odot v_{1}\right) \ominus\left(u_{1} \odot v_{2}\right)\right](e)}{\left[\left(u_{2} \odot v_{1}\right) \ominus\left(u_{1} \odot v_{2}\right)\right](1)}=1,
$$

and

$$
\int_{1}^{e}\left[u^{T}\left(t, \lambda_{1}\right) \odot v\left(t, \lambda_{2}\right)\right]^{d t}=1
$$


where $\lambda_{1} \neq \lambda_{2}$. Here, we have used the below matrix circle product:

$$
\begin{aligned}
u^{T}\left(t, \lambda_{1}\right) \odot v\left(t, \lambda_{2}\right) & =\left(u_{1}\left(t, \lambda_{1}\right) u_{2}\left(t, \lambda_{1}\right)\right) \odot\left(\begin{array}{c}
v_{1}\left(t, \lambda_{2}\right) \\
v_{2}\left(t, \lambda_{2}\right)
\end{array}\right) \\
& =\left[\left(u_{1}\left(t, \lambda_{1}\right) \odot v_{1}\left(t, \lambda_{2}\right)\right) \oplus\left(u_{2}\left(t, \lambda_{1}\right) \odot v_{2}\left(t, \lambda_{2}\right)\right)\right] .
\end{aligned}
$$

Lemma 3.2. All eigenvalues of the multiplicative Dirac system (1)-(2) are real.

Proof: Let $\lambda_{1}=u+i v$ and $\overline{\lambda_{1}}=u-i v$. By the definition of orthogonality, we can write

$$
\int_{1}^{e}\left\{\left[\left(y_{1}\left(x, \lambda_{1}\right) \odot \overline{y_{1}\left(x, \lambda_{1}\right)}\right) \oplus\left(y_{2}\left(x, \lambda_{1}\right) \odot \overline{y_{2}\left(x, \lambda_{1}\right)}\right)\right]^{\lambda_{1}-\overline{\lambda_{1}}}\right\}^{d x}=1 .
$$

Since $\lambda_{1} \neq \overline{\lambda_{1}}$, we get

$$
\int_{1}^{e}\left[\left(y_{1}\left(x, \lambda_{1}\right) \odot \overline{y_{1}\left(x, \lambda_{1}\right)}\right) \oplus\left(y_{2}\left(x, \lambda_{1}\right) \odot \overline{y_{2}\left(x, \lambda_{1}\right)}\right)\right]^{d x}=1 .
$$

So, by using the circular product and conjugate operations,

$$
\begin{aligned}
\int_{1}^{e}\left[\left(y_{1}\left(x, \lambda_{1}\right) \odot \overline{y_{1}\left(x, \lambda_{1}\right)}\right) \oplus\left(y_{2}\left(x, \lambda_{1}\right) \odot \overline{y_{2}\left(x, \lambda_{1}\right)}\right)\right]^{d x} & =e^{\int^{e} \ln \left(y_{1}^{\ln \overline{y_{1}\left(x, \lambda_{1}\right)}} y_{2}^{\ln \overline{y_{2}\left(x, \lambda_{1}\right)}}\right) d x} \\
& =e^{\int^{e}\left(\left|\ln y_{1}\right|^{2}+\left|\ln y_{2}\right|^{2}\right) d x} .
\end{aligned}
$$

By (7),

$$
\int_{1}^{e}\left(\left|\ln y_{1}\right|^{2}+\left|\ln y_{2}\right|^{2}\right) d x=0 \Rightarrow y_{1}=1, y_{2}=1 .
$$

This is a contradiction. It is due to our assumption. So the chosen eigenvalue is real. Since this eigenvalue is arbitrary, all eigenvalues of the problem are real. We can prove this lemma with another method. Let's express this proof now.

Assume that $\lambda_{0}$ is a complex eigenvalue corresponding eigenfunction $\left(\overline{y_{1}}(x), \overline{y_{2}}(x)\right)^{T}$. By using the properties of *derivative, we get

$$
\left[\left(y_{2} \odot \overline{y_{1}}\right) \ominus\left(y_{1} \odot \overline{y_{2}}\right)\right]^{*}=\left[\left(y_{1} \odot \overline{y_{1}}\right) \oplus\left(y_{2} \odot \overline{y_{2}}\right)\right]^{\lambda_{0}-\overline{\lambda_{0}}} .
$$

By *integration on $[1, e]$, since

$$
\int_{1}^{e}\left\{\left[\left(y_{1} \odot \overline{y_{1}}\right) \oplus\left(y_{2} \odot \overline{y_{2}}\right)\right]^{\lambda_{0}-\overline{\lambda_{0}}}\right\}^{d x}=1
$$

we have

$$
\int_{1}^{e}\left(\left|\ln y_{1}\right|^{2}+\left|\ln y_{2}\right|^{2}\right) d x=0 \Rightarrow y_{1}=1, y_{2}=1 .
$$

This is also a contradiction as just happened. Therefore, the considered eigenvalue is real.

Now let us examine another important spectral property of this problem. The simplicity of eigenvalues is a very important feature in mathematical physics and there are many proof techniques for simplicity. The algebraic multiplicity of an eigenvalue is the number of times it repeats as a root of the characteristic polynomial. If the algebraic multiplicity of an eigenvalue is 1 , that eigenvalue is called a simple eigenvalue. 
Lemma 3.3. All eigenvalues of the multiplicative Dirac system (1)-(2) are simple.

Proof: Let $\lambda, \mu \in \mathbb{C}$ and $\lambda \neq \mu$ be eigenvalues of the given problem. Then, we get

$\left\{\left[y_{1}(x, \lambda) \odot y_{2}(x, \mu)\right] \ominus\left[y_{2}(x, \lambda) \odot y_{1}(x, \mu)\right]\right\}^{*}=\left\{\left[y_{1}(x, \lambda) \odot y_{1}(x, \mu)\right] \oplus\left[y_{2}(x, \lambda) \odot y_{2}(x, \mu)\right]\right\}^{\mu-\lambda}$

By the *integration on $[1, e]$,

$\int_{1}^{e}\left\{\left[\left(y_{1}(x, \lambda) \odot y_{1}(x, \mu)\right) \oplus\left(y_{2}(x, \lambda) \odot y_{2}(x, \mu)\right)\right]^{\mu-\lambda}\right\}^{d x}=\left[y_{1}(e, \lambda) \odot y_{2}(e, \mu)\right] \ominus\left[y_{2}(e, \lambda) \odot y_{1}(e, \mu)\right]$,

and after some adjustments are made here, we get

$e^{\mu-\lambda} \odot \int_{1}^{e}\left[\left(y_{1}(x, \lambda) \odot y_{1}(x, \mu)\right) \oplus\left(y_{2}(x, \lambda) \odot y_{2}(x, \mu)\right)\right]^{d x}=\left[y_{1}(e, \lambda) \odot y_{2}(e, \mu)\right] \ominus\left[y_{2}(e, \lambda) \odot y_{1}(e, \mu)\right]$.

Here, if the integral to the left of the equation is left alone,

$\int_{1}^{e}\left[\left(y_{1}(x, \lambda) \odot y_{1}(x, \mu)\right) \oplus\left(y_{2}(x, \lambda) \odot y_{2}(x, \mu)\right)\right]^{d x}=e^{\frac{1}{\mu-\lambda}} \odot\left\{\left[y_{1}(e, \lambda) \odot y_{2}(e, \mu)\right] \ominus\left[y_{2}(e, \lambda) \odot y_{1}(e, \mu)\right]\right\}$.

Then, if limit operation is applied to both sides of above relation as $\mu \rightarrow \lambda$, it yields

$$
e^{\int^{e}\left(\left|\ln y_{1}(x, \lambda)\right|^{2}+\left|\ln y_{2}(x, \lambda)\right|^{2}\right) d x}=e^{\frac{\partial \ln y_{2}(e, \lambda)}{\partial \lambda} \ln y_{1}(e, \lambda)-\frac{\partial \ln y_{1}(e, \lambda)}{\partial \lambda} \ln y_{2}(e, \lambda),}
$$

by multiplicative L'Hopital's rule. Hence,

$$
\Lambda(\lambda)=\left[e^{\gamma} \odot y_{1}(e, \lambda)\right] \oplus\left[e^{\delta} \odot y_{2}(e, \lambda)\right]
$$

has only simple zeros. Indeed, conversely, assume that $\widetilde{\lambda}$ is double-decker root of $\Lambda(\lambda)$. So,

$$
\left[e^{\gamma} \odot y_{1}(e, \tilde{\lambda})\right] \oplus\left[e^{\delta} \odot y_{2}(e, \tilde{\lambda})\right]=1 .
$$

Here, the applying * partial derivative to both sides gives

$$
\left[e^{\gamma} \odot \frac{\partial^{*} y_{1}(e, \tilde{\lambda})}{\partial \lambda}\right] \oplus\left[e^{\delta} \odot \frac{\partial^{*} y_{2}(e, \tilde{\lambda})}{\partial \lambda}\right]=1 .
$$

So, from (9) and (10),

$$
\left[y_{1}(e, \tilde{\lambda}) \odot \frac{\partial^{*} y_{2}(e, \tilde{\lambda})}{\partial \lambda}\right] \ominus\left[y_{2}(e, \tilde{\lambda}) \odot \frac{\partial^{*} y_{1}(e, \tilde{\lambda})}{\partial \lambda}\right]=1 .
$$

If the basic properties of multiplicative analysis are used in (11), we get

$$
\frac{y_{1}(e, \widetilde{\lambda})^{\frac{\partial}{\partial \lambda} \ln y_{2}(e, \widetilde{\lambda})}}{y_{2}(e, \widetilde{\lambda})^{\frac{\partial}{\partial \lambda} \ln y_{1}(e, \widetilde{\lambda})}}=1,
$$

and

$$
\frac{\partial \ln y_{2}(e, \lambda)}{\partial \lambda} \ln y_{1}(e, \lambda)-\frac{\partial \ln y_{1}(e, \lambda)}{\partial \lambda} \ln y_{2}(e, \lambda)=0 .
$$


By considering (8), since

$$
\int_{1}^{e}\left(\left|\ln y_{1}(x, \widetilde{\lambda})\right|^{2}+\left|\ln y_{2}(x, \widetilde{\lambda})\right|^{2}\right) d x=0
$$

we have

$$
y_{1}(x, \tilde{\lambda})=1=y_{2}(x, \tilde{\lambda})
$$

for $\lambda=\widetilde{\lambda}$. This is a contradiction. Therefore, $\Lambda(\lambda)$ has only simple zeros. It completes the proof.

Lemma 3.4. The multiplicative Dirac operator $L$ in (1) is formally self-adjoint on $L_{2}^{*}[1, e]$.

Proof: Let us use the definition of inner product on $L_{2}^{*}[1, e]$ and *integration.

$$
\begin{aligned}
& <L u, v>_{*}=\int_{1}^{e}\left[(L u)^{T} \odot v\right]^{d t}=\int_{1}^{e}\left[\left(u_{2}^{*} u_{1}^{q(t)}\left(u_{1}^{*}\right)^{-1} u_{2}^{r(t)}\right) \odot\left(\begin{array}{c}
v_{1} \\
v_{2}
\end{array}\right)\right]^{d t} \\
& =\int_{1}^{e}\left[\left(\left(u_{2}^{*} u_{1}^{q(t)}\right) \odot v_{1}\right) \oplus\left(\left(\left(u_{1}^{*}\right)^{-1} u_{2}^{r(t)}\right) \odot v_{2}\right)\right]^{d t}
\end{aligned}
$$

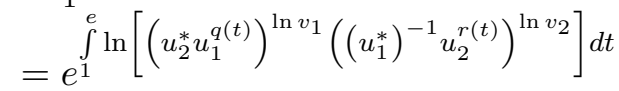

$$
\begin{aligned}
& =e^{\int_{1}^{e}\left[\ln \left(u_{2}^{*}\right) \ln v_{1}+\ln v_{1} \ln u_{1}^{q(t)}-\ln \left(u_{1}^{*}\right) \ln v_{2}+\ln v_{2} \ln u_{2}^{r(t)}\right] d t} .
\end{aligned}
$$

If the integration by parts formula and the relation between multiplicative and usual derivatives are considered some basic features of multiplicative calculus are used after some algebraic operations and simplifications, it yields

$$
\begin{aligned}
<L u, v>_{*} & =e^{\ln \left[\left(\left(u_{2} \odot v_{1}\right) \ominus\left(u_{1} \odot v_{2}\right)\right)(e) \ominus\left(\left(u_{2} \odot v_{1}\right) \ominus\left(u_{1} \odot v_{2}\right)\right)(1)\right]} \\
& =e^{\int^{e} \ln \left[\left(v_{2}^{*} v_{1}^{q(t)}\right)^{\ln u_{1}}\left(\left(v_{1}^{*}\right)^{-1} v_{2}^{r(t)}\right)^{\ln u_{2}}\right] d t} \\
& =e^{\ln \left[W_{m}(u, v)(e) \ominus W_{m}(u, v)(1)\right]+\int_{1}^{e} \ln \left[(L v)^{T} \odot u\right] d t} \\
& =W_{m}(u, v)(e) \ominus W_{m}(u, v)(1) \oplus \int_{1}^{e}\left[(L v)^{T} \odot u\right]^{d t}=<u, L v>_{*},
\end{aligned}
$$

where $W_{m}(u, v)=\left(u_{2} \odot v_{1}\right) \ominus\left(u_{1} \odot v_{2}\right)$. It completes the proof.

Self-adjoint operators are used in functional analysis and quantum mechanics. In quantum mechanics their importance lies in the Dirac-von Neumann formulation of quantum mechanics, in which physical observables such as position, momentum, angular momentum and spin are represented by self-adjoint operators.

Example 3.5. Let us consider the below multiplicative Dirac system

$$
\begin{aligned}
& y_{2}^{*} y_{1}^{c}=y_{1}^{\lambda}, \\
& y_{1}^{*} y_{2}^{-d}=y_{2}^{-\lambda},
\end{aligned}
$$

which is obtained by setting $q(t)=c \in \mathbb{R}, r(t)=d \in \mathbb{R}$ in (1) with the initial conditions

$$
y_{1}(0)=e^{\alpha}, y_{2}(0)=e^{\beta}
$$

The system (12) is actually equivalent to the below non-linear system

$$
\begin{aligned}
& y_{2}^{\prime}+c y_{2} \ln y_{1}=\lambda y_{2} \ln y_{1}, \\
& -y_{1}^{\prime}+d y_{1} \ln y_{2}=\lambda y_{1} \ln y_{2}
\end{aligned}
$$


in usual case.

$$
y_{1}(t)= \begin{cases}e^{\alpha \cos \sqrt{k} t-\frac{\beta(\lambda-d)}{\sqrt{k}} \sin \sqrt{k} t}, & k>0 \\ e^{\alpha-\beta(\lambda-d) t}, & k=0 \\ e^{\alpha \cosh \sqrt{-k} t-\frac{\beta(\lambda-d)}{\sqrt{-k}} \sinh \sqrt{-k} t}, & k<0\end{cases}
$$

and

$$
y_{2}(t)= \begin{cases}e^{\frac{\alpha(\lambda-c)}{\sqrt{k}} \sin \sqrt{k} t+\beta \cos \sqrt{k} t}, & k>0 \\ e^{\alpha(\lambda-c) t+\beta}, & k=0 \\ e^{\frac{\alpha(\lambda-c)}{\sqrt{-k}} \sinh \sqrt{-k} t+\beta \cosh \sqrt{-k} t}, & k<0\end{cases}
$$

are the eigenfunctions of the problem (12)-(13) where $k=(\lambda-c)(\lambda-d)$. It can be easily shown that these solutions also coincide with the solutions of the problem (14)-(13). This example shows how effective multiplicative analysis is in solving problems in the classical case.

\section{Conclusion}

In this study, we defined a multiplicative Dirac system and obtained the eigenfunctions of the problem. Later, we examined this important problem in the multiplicative case in terms of spectral analysis. We think that this system, which is extremely important for quantum physics and effective in the classical case, will make great contributions to mathematical physics in the multiplicative case. In fact, this equation, which we examined in the multiplicative case, corresponds to an equation that is much more difficult and tiring to analyze in classical a nalysis. This situation increases the importance of the results we obtained and the different analysis we used.

\section{References}

Akkus, I., \& Kizilaslan, G. (2019). Quaternions: Quantum calculus approach with applications. Kuwait Journal of Science, 46(4), 1-13.

Allahverdiev, B. P., \& Tuna, H. (2017). One dimensional $q$ - Dirac equation. Mathematical Methods in the Applied Sciences, 40, 7287-7306.

Bairamov, E., Aygar, Y., \& Olgun, M. (2010). Jost solution and the spectrum of the discrete Dirac systems. Boundary Value Problems, 306571, 1-11.

Bashirov, A. E., Kurpınar, E. M., \& Özyapıcı, A. (2008). Multiplicative calculus and its applications. Journal of Mathematical Analysis and Applications, 337, 36-48.

Bashirov, A. E., Mısırlı E., Tandogdu, Y., \& Özyapıcı, A. (2011). On modeling with multiplicative differential equations. Applied Mathematics-A Journal of Chinese Universities, 26(4), 425-438.

Bashirov, A. E., \& Norozpur, S. (2018). On an alternative view to complex calculus. Mathematical Methods in Applied Sciences, 41, 7313-7324.

Bashirov, A. E., \& Riza, M. (2011). On complex multiplicative differentiation. TWMS Journal on Applied and Engineering Mathematics, 1(1), 75-85.

Benford, A. (1938). The Law of anomalous numbers. Proceedings of the American Philosophical Society, $78,551-572$.

Boruah, K., \& Hazarika, B. (2018). G-Calculus. TWMS Journal of Applied and Engineering Mathematics, 8(1), 94-105

Çöl A., \& Mamedov Kh. R. (2012). On an inverse scattering problem for a class of Dirac operators with spectral parameter in the boundary condition. Journal of Mathematical Analysis and Applications, 393(2), 470-478. 
Dirac, P. A. M. (1928). The quantum theory of the electron. Proceedings of the Royal Society A: Mathematical, Physical and Engineering Sciences, 117, 610-624.

Florack, L., \& Assen, Hv. (2012). Multiplicative Calculus in Biomedical Image Analysis. Journal of Mathematical Imaging and Vision, 42, 64-75.

Gasymov, M. G., \& Levitan, B. M. (1966). The inverse problem for the Dirac system. Doklady Akademy Nauk SSSR, 167(6), 967-970.

Grossman, M. (1979). An introduction to Non-Newtonian calculus.International Journal of Mathematical Education in Science and Technology, 10(4), 525-528.

Grossman, M., \& Katz, R. (1972). Non-Newtonian calculus. Pigeon Cove, MA: Lee Press.

Guenther, R. A. (1983). Product integrals and sum integrals. International Journal of Mathematical Education in Science and Technology, 14(2), 243-249.

Gurefe, Y., Kadak, U., Mısırlı, E., \& Kurdi, A. (2016). A new look at the classical sequence spaces by using multiplicative calculus. UPB Scientific Bulletin, Series A, 78(2), 9-20.

Joa, I., \& Minkin, A. (1997). Eigenfunction estimate for a Dirac operator. Acta Mathematica Hungarica, 76(4), 337-349.

Kadak, U., \& Gurefe, Y. (2016). A generalization on weighted means and convex functions with respect to the Non-Newtonian calculus. International Journal of Analysis, Article ID 5416751, 1-9.

Keskin, B., \& Ozkan, A. S. (2011). Inverse spectral problems for Dirac operator with eigenvalue dependent boundary and jump conditions. Acta Mathematica Hungarica, 130(4), 309-320.

Levitan, B.M., \& Sargsjan, I.S. (1991). Sturm-Liouville and Dirac operators. Moscow: Nauka.

Moses, H. E. (1956). Calculation of the scattering potential from reflection coefficients. Physical Review, 102(2), 559-567.

Prats, F., \& Toll J. S. (1959). Construction of the Dirac equation central potential from phase shifts and bound states. Physical Review, 113(1), 363-370.

Sharma, L. K., Luhanga, P. V., \& Chimidza, S. (2011). Potentials for the Klein-Gordon and Dirac equations. Chiang Mai Journal of Science, 38(4), 514-526.

Slavík, A. (2007). Product Integration, Its History and Applications. Prague: Matfyzpress.

Stanley, D. (1999). A multiplicative calculus. Primus, 9(4), 310-326.

Thaller, B. (1992). The Dirac Equation. New York: Springer-Verlag.

Verde, M. (1959). The inversion problem in wave mechanics and dispersion relations. Nuclear Physics, $9(2), 255-266$.

Weiss, R., Stahel, W., \& Scharf, G. (1972). The inverse problem of potential scattering according to the Dirac equation. Nuclear Physics A, 183(2), 337-351.

Yazlık, Y., Tollu, D. T., \& Taşkara, N. (2016). On the solutions of a three-dimensional system of difference equations. Kuwait Journal of Science, 43(1), 95-111.

Submitted: $\quad 07 / 04 / 2021$

Revised: $\quad 13 / 07 / 2021$

Accepted: $\quad 15 / 08 / 2021$

DOI: $\quad 10.48129 / \mathrm{kjs} .13411$ 